\title{
POSITIVE CONJUGACY CLASSES IN WEYL GROUPS
}

\author{
GEORGE LUSZTIG
}

Department of Mathematics, M.I.T., Cambridge, MA 02139, USA.

E-mail: gyuri@math.mit.edu

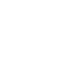

1. Let $W$ be a Weyl group. In this paper we introduce the notion of positive conjugacy class of $W$. This generalizes the notion of elliptic regular conjugacy class in the sense of Springer [9].

Let $w \mapsto|w|$ be the length function on $W$. Let $S=\{s \in W ;|s|=1\}$.

Let $v$ be an indeterminate. Recall that the Iwahori-Hecke algebra of $W$ is the associative $\mathbf{Q}(v)$-algebra $H$ which, as a $\mathbf{Q}(v)$-vector space has basis $\left\{T_{w} ; w \in W\right\}$ and has multiplication given by $T_{w} T_{w^{\prime}}=T_{w w^{\prime}}$ if $\left|w w^{\prime}\right|=$ $|w|+\left|w^{\prime}\right|$ and $\left(T_{s}+1\right)\left(T_{s}-v^{2}\right)=0$ if $s \in S$; note that $T_{1}$ is the unit element of $H$. This is a split semisimple algebra. Let $\mathbf{q}=v^{2}$.

For $w, w^{\prime}$ in $W$ let $N^{w, w^{\prime}}$ be the trace of the $\mathbf{Q}(v)$-linear map $H \rightarrow H$, $h \mapsto T_{w} h T_{w^{\prime-1}}$. We have $N^{w, w^{\prime}} \in \mathbf{Z}[\mathbf{q}]$ and

$$
N^{w, w^{\prime}}=\sum_{E \in \operatorname{Irr} W} \operatorname{tr}\left(T_{w}, E_{v}\right) \operatorname{tr}\left(T_{w^{\prime}}, E_{v}\right)
$$

where $\operatorname{Irr} W$ is the set of irreducible $\mathbf{Q}[W]$-modules up to isomorphism and for $E \in \operatorname{Irr} W, E_{v}$ denotes the corresponding simple $H$-module (which in 8, 3.3] is denoted by $\left.E\left(v^{2}\right)\right)$. Note that when $v$ is specialized to $1, H$ becomes the group algebra $\mathbf{Q}[W]$ of $W$ and $N^{w, w^{\prime}}$ specializes to $N^{w, w^{\prime}}(1)$, the number of elements $y \in W$ such that $w y=y w^{\prime}$. In particular, if $w \in W$, $N^{w, w}$ specializes to $n^{w}$, the order of the centralizer of $w$ in $W$; thus the polynomial $N^{w, w}$ can be viewed as a q-analogue of the number $n^{w}$.

Received October 5, 2020 and in revised form December 22, 2020.

AMS Subject Classification: 20G99.

Key words and phrases: Weyl group, Hecke algebra, conjugacy class.

Supported by NSF grant DMS-1566618. 
If $C$ is a conjugacy class in $W$ we denote by $C_{\text {min }}$ the set of all $y \in C$ such that $C \rightarrow \mathbf{N}, w \mapsto|w|$, reaches its minimum at $y$. By a result of Geck and Pfeiffer [4, 3.2.9], for any $E \in \operatorname{Irr} W, w \mapsto \operatorname{tr}\left(T_{w}, E_{v}\right)$ is constant on $C_{\text {min }}$. Using this and (a) we see that $w \mapsto N^{w, w}$ is constant on $C_{\text {min }}$. We say that $C$ is positive if $C \neq\{1\}$ and for some/any $w \in C_{\min }$ we have $N^{w, w} \in \mathbf{N}[\mathbf{q}]$. (We then also say that any element $w \in C_{\min }$ is positive.)

2. For any $f \in \mathbf{Z}[\mathbf{q}]$ we write $f=\sum_{i>0} f_{i} \mathbf{q}^{i}$ where $f_{i} \in \mathbf{Z}$. For $w \in W$ let $S_{w}$ be the set of all $s \in S$ such that $s$ appears in some/any reduced expression for $w$. Let $\mathcal{L}_{w}=\{s \in S ;|s w|<|w|\}, \mathcal{R}_{w}=\{s \in S ;|w s|<|w|\}$. For $a, a^{\prime}$ in $W$ we write $T_{a} T_{a^{\prime}}=\sum_{b \in W} \phi\left(a, a^{\prime}, b\right) T_{b}$ where $\phi\left(a, a^{\prime}, b\right) \in \mathbf{Z}[\mathbf{q}]$. We show:

(a) $\phi\left(a, a^{\prime}, b\right)_{|a|} \geq 0, \phi\left(a, a^{\prime}, b\right)_{i}=0$ for $i>|a|$. If $\phi\left(a, a^{\prime}, b\right)_{|a|} \neq 0$ then either $a^{\prime}=b, S_{a} \subset \mathcal{L}_{a^{\prime}}$ or $|b|<\left|a^{\prime}\right|$. If $a^{\prime}=b, S_{a} \subset \mathcal{L}_{a^{\prime}}$ then $\phi\left(a, a^{\prime}, b\right)_{|a|}=1$.

We argue by induction on $|a|$. When $|a|=0$ the result is obvious. Assume now that $|a| \geq 1$. We write $a=a_{1} s$ where $s \in S,\left|a_{1}\right|=|a|-1$. If $\left|s a^{\prime}\right|=\left|a^{\prime}\right|+1$ then $\phi\left(a, a^{\prime}, b\right)=\phi\left(a_{1}, s a^{\prime}, b\right)$ and the induction hypothesis shows that $\phi\left(a, a^{\prime}, b\right)_{i}=0$ for $i \geq|a|$. Since $s \in S_{a}, s \notin \mathcal{L}_{a^{\prime}}$, we see that the desired result holds. Next we assume that $\left|s a^{\prime}\right|=\left|a^{\prime}\right|-1$. Then $\phi\left(a, a^{\prime}, b\right)=\mathbf{q} \phi\left(a_{1}, s a^{\prime}, b\right)+(\mathbf{q}-1) \phi\left(a_{1}, a^{\prime}, b\right)$. From the induction hypothesis we see that $\phi\left(a, a^{\prime}, b\right)_{i}=0$ if $i>|a|$, that $\phi\left(a, a^{\prime}, b\right)_{|a|}=\phi\left(a_{1}, s a^{\prime}, b\right)_{\left|a_{1}\right|}+$ $\phi\left(a_{1}, a^{\prime}, b\right)_{\left|a_{1}\right|} \geq 0$ and that if $\phi\left(a, a^{\prime}, b\right)_{|a|} \neq 0$ then either $\phi\left(a_{1}, s a^{\prime}, b\right)_{\left|a_{1}\right|} \neq 0$ or $\phi\left(a_{1}, a^{\prime}, b\right)_{\left|a_{1}\right|} \neq 0$, so that we are in one of the cases (i)-(iv) below.

(i) $s a^{\prime}=b$,

(ii) $|b|<\left|s a^{\prime}\right|$;

(iii) $a^{\prime}=b, S_{a_{1}} \subset \mathcal{L}_{a^{\prime}}$;

(iv) $|b|<\left|a^{\prime}\right|$.

In case (i), (ii), (iii) we have $|b|<\left|a^{\prime}\right|$; in case (iii) have $a^{\prime}=b$ and $S_{a} \subset \mathcal{L}_{a^{\prime}}$ (since $S_{a}=S_{a_{1}} \cup\{s\}$ ), as desired. Now assume that $a^{\prime}=b$, $S_{a} \subset \mathcal{L}_{a^{\prime}}$. It remains to show that $\phi\left(a_{1}, s a^{\prime}, b\right)_{\left|a_{1}\right|}+\phi\left(a_{1}, a^{\prime}, b\right)_{\left|a_{1}\right|}=1$. By the induction hypothesis we have $\phi\left(a_{1}, a^{\prime}, b\right)_{\left|a_{1}\right|}=1$ (since $S_{a_{1}} \subset \mathcal{L}_{a^{\prime}}$ ) $\phi\left(a_{1}, s a^{\prime}, b\right)_{\left|a_{1}\right|}=0$ (since $s a^{\prime} \neq b$ and $\left.|b| \nless\left|s a^{\prime}\right|\right)$. This completes the proof. 
The following result is proved in the same way as (a).

(b) $\phi\left(a, a^{\prime}, b\right)_{\left|a^{\prime}\right|} \geq 0, \phi\left(a, a^{\prime}, b\right)_{i}=0$ for $i>\left|a^{\prime}\right|$. If $\phi\left(a, a^{\prime}, b\right)_{\left|a^{\prime}\right|} \neq 0$ then either $a=b, S_{a^{\prime}} \subset \mathcal{R}_{a^{\prime}}$ or $|b|<|a|$. If $a=b, S_{a^{\prime}} \subset \mathcal{R}_{a^{\prime}}$ then $\phi\left(a, a^{\prime}, b\right)_{\left|a^{\prime}\right|}=1$. For $a, a^{\prime}, a^{\prime \prime}$ in $W$ we have $T_{a} T_{a^{\prime}} T_{a^{\prime \prime}}=\sum_{b \in W} f\left(a, a^{\prime}, a^{\prime \prime}, b\right) T_{b}$ where $f\left(a, a^{\prime}, a^{\prime \prime}, b\right) \in \mathbf{Z}[\mathbf{q}]$. Let $n=|a|+\left|a^{\prime \prime}\right|$. We show:

(c) $f\left(a, a^{\prime}, a^{\prime \prime}, a^{\prime}\right)_{n} \geq 0, f\left(a, a^{\prime}, a^{\prime \prime}, a^{\prime}\right)_{i}=0$ for $i>n$. If $f\left(a, a^{\prime}, a^{\prime \prime}, a^{\prime}\right)_{n} \neq 0$ then $S_{a} \subset \mathcal{L}_{a^{\prime}}$ and $S_{a^{\prime \prime}} \subset \mathcal{R}_{a^{\prime}}$. Conversely, if $S_{a} \subset \mathcal{L}_{a^{\prime}}$ and $S_{a^{\prime \prime}} \subset \mathcal{R}_{a^{\prime}}$ then $f\left(a, a^{\prime}, a^{\prime \prime}, a^{\prime}\right)_{n}=1$.

We have $f\left(a, a^{\prime}, a^{\prime \prime}, a^{\prime}\right)=\sum_{c \in W} \phi\left(a, a^{\prime}, c\right) \phi\left(c, a^{\prime \prime}, a^{\prime}\right)$. Hence for $i \geq 0$ we have $f\left(a, a^{\prime}, a^{\prime \prime}, a^{\prime}\right)_{i}=\sum_{c \in W ; j \geq 0, j^{\prime} \geq 0, j+j^{\prime}=i} \phi\left(a, a^{\prime}, c\right)_{j} \phi\left(c, a^{\prime \prime}, a^{\prime}\right)_{j^{\prime}}$. Using (a) and (b) in the last sum we can take $j \geq|a|, j^{\prime} \geq\left|a^{\prime \prime}\right|$. Hence if $i>n=$ $|a|+\left|a^{\prime \prime}\right|$ then $f\left(a, a^{\prime}, a^{\prime \prime}, a^{\prime}\right)_{i}=0$ and

$f\left(a, a^{\prime}, a^{\prime \prime}, a^{\prime}\right)_{n}=\sum_{c \in W} \phi\left(a, a^{\prime}, c\right)_{|a|} \phi\left(c, a^{\prime \prime}, a^{\prime}\right)_{\left|a^{\prime \prime}\right|} \geq 0$. Assume now that $f\left(a, a^{\prime}, a^{\prime \prime}, a^{\prime}\right)_{n} \neq 0$. Then in the last sum we can assume that

$$
c=a^{\prime}, S_{a} \subset \mathcal{L}_{a^{\prime}} \text { or }|c|<\left|a^{\prime}\right| \text { and } a^{\prime}=c, S_{a^{\prime \prime}} \subset \mathcal{R}_{c} \text { or }\left|a^{\prime}\right|<|c|
$$

Thus we can assume that $c=a^{\prime}, S_{a} \subset \mathcal{L}_{a^{\prime}}$ and $S_{a^{\prime \prime}} \subset \mathcal{R}_{a^{\prime}}$ and using again (a), (b) we have $f\left(a, a^{\prime}, a^{\prime \prime}, a^{\prime}\right)_{n}=1$.

For $w, w^{\prime}$ in $W$ we set $n=|w|+\left|w^{\prime}\right|$; we show:

(d) $N_{n}^{w, w^{\prime}}=\sharp\left(a^{\prime} \in W ; S_{w} \subset \mathcal{L}_{a^{\prime}}, S_{w^{\prime}} \subset \mathcal{R}_{a^{\prime}}\right)>0, N_{i}^{w, w^{\prime}}=0$ for $i>n$.

We have $N^{w, w^{\prime}}=\sum_{a^{\prime} \in W} f\left(w, a^{\prime}, w^{\prime}, a^{\prime}\right)$ and the result follows from (c). (We use that if $a^{\prime}=w_{0}$, the longest element of $W$ then $S_{w} \subset \mathcal{L}_{a^{\prime}}=S$, $S_{w^{\prime}} \subset \mathcal{R}_{a^{\prime}}=S$.)

From (d) we deduce:

(e) Let $w, w^{\prime}, n$ be as in (d). Assume that either $S_{w}=S$ or $S_{w^{\prime}}=S$. then $N_{n}^{w, w^{\prime}}=1$.

Indeed, if $a^{\prime} \in W$ satisfies $S \subset \mathcal{L}_{a^{\prime}}$ or $S \subset \mathcal{R}_{a^{\prime}}$ then $a^{\prime}=w_{0}$.

We state the following result.

(f) Let $w, w^{\prime}, n$ be as in (d). For $i=0,1, \ldots, n$ we have $N_{i}^{w, w^{\prime}}=(-1)^{n} N_{n-i}^{w, w^{\prime}}$. Let $^{-}: \mathbf{Q}(v) \rightarrow \mathbf{Q}(v)$ be the field automorphism such that $\bar{v}=v^{-1}$. For $E \in$ $\operatorname{Irr} W$ let $E^{\dagger} \in \operatorname{Irr} W$ be the tensor product of $E$ with the sign representation of $W$. It is known that for $w \in W$ we have

$$
\operatorname{tr}\left(T_{w}, E_{v}^{\dagger}\right)=\left(-v^{2}\right)^{|w|} \operatorname{tr}\left(T_{w^{-1}}^{-1}, E_{v}\right)=\left(-v^{2}\right)^{|w|} \overline{\operatorname{tr}\left(T_{w}, E_{v}\right)} .
$$


It follows that

$$
\begin{aligned}
N^{w, w^{\prime}} & =\sum_{E \in \operatorname{Irr} W} \operatorname{tr}\left(T_{w}, E_{v}^{\dagger}\right) \operatorname{tr}\left(T_{w^{\prime}}, E_{v}^{\dagger}\right) \\
& =\sum_{E \in \operatorname{Irr} W}\left(-v^{2}\right)^{|w|} \overline{\operatorname{tr}\left(T_{w}, E_{v}\right)}\left(-v^{2}\right)^{\left|w^{\prime}\right|} \overline{\operatorname{tr}\left(T_{w^{\prime}}, E_{v}\right)} \\
& =\sum_{E \in \operatorname{Irr} W}\left(-v^{2}\right)^{n} \overline{\operatorname{tr}\left(T_{w}, E_{v}\right) \operatorname{tr}\left(T_{w^{\prime}}, E_{v}\right)} .
\end{aligned}
$$

We see that

$$
N^{w, w^{\prime}}=(-\mathbf{q})^{n} \overline{N^{w, w^{\prime}}}
$$

and (f) follows.

3. We now assume that $W$ is irreducible. Let $\nu=\left|w_{0}\right|$ where $w_{0}$ is the longest element of $W$. An element $w \in W$ (or its conjugacy class) is said to be elliptic if its eigenvalues in the reflection representation of $W$ are all $\neq 1$. For any $d \in\{2,3,4, \ldots\}$ let $C^{d}$ be the set of all elliptic elements $w \in W$ which have order $d$ and are regular in the sense of Springer [9]. It is known [9] that $C^{d}$ is either empty or a single conjugacy class in $W$. Let $\mathcal{D}=\left\{d \in\{2,3, \ldots\} ; C^{d} \neq \emptyset\right\}$. It is known [9] that if $d \in \mathcal{D}$ and $w \in C_{\text {min }}^{d}$ then $|w|=2 \nu / d$. Let $h$ be the Coxeter number of $W$. We have $h \in \mathcal{D}$.

According to [9], the set $\mathcal{D}$ is as follows:

Type $A_{n}(n \geq 1): \mathcal{D}=\{n+1\}$.

Type $B_{n}(n \geq 2): \mathcal{D}=\{d \in\{2,4,6, \ldots\} ; 2 n / d=$ integer $\}$.

Type $D_{n}(n$ even, $n \geq 4)$ :

$\mathcal{D}=\{d \in\{2,4,6, \ldots\} ;(2 n-2) / d=$ odd integer or $2 n / d=$ integer $\}$.

Type $D_{n}(n$ odd, $n \geq 5): \mathcal{D}=\{d \in\{2,4,6, \ldots\} ;(2 n-2) / d=$ odd integer $\}$.

Type $E_{6}: \mathcal{D}=\{3,6,9,12\}$.

Type $E_{7}: \mathcal{D}=\{2,6,14,18\}$.

Type $E_{8}: \mathcal{D}=\{2,3,4,5,6,8,10,12,15,20,24,30\}$.

Type $F_{4}: \mathcal{D}=\{2,3,4,6,8,12\}$.

Type $G_{2}: \mathcal{D}=\{2,3,6\}$. 
We note the following properties:

(a) If $2 \in \mathcal{D}, d \in \mathcal{D}$ is even and $w \in C_{\text {min }}^{d}$ then $w^{d / 2}=w_{0},(d / 2)|w|=\left|w_{0}\right|$ hence $T_{w}^{d / 2}=T_{w_{0}}$.

(b) If $d=h, w \in C_{\text {min }}^{d}$ then $T_{w}^{d}=T_{w_{0}}^{2}$.

(c) If $d \in \mathcal{D}, h / d \in \mathbf{N}$ and $y \in C_{\text {min }}^{h}$ then $y^{h / d} \in C_{\text {min }}^{d}$ and $(h / 2)|y|=\left|y^{h / d}\right|$ hence $T_{y}^{h / d}=T_{y^{h / d}}$.

The equation $w^{d / 2}=w_{0}$ in (a) holds by examining the characteristic polynomial of $w$ and $w^{d / 2}$ in the reflection representation of $W$; then (a) follows. The equality in (b) can be deduced from [1, Ch.V, $\S 6$, Ex.2]. The equation $w^{h / d} \in C_{\min }^{d}$ in (c) holds by examining the characteristic polynomial of $w$ and $w^{h / d}$ in the reflection representation of $W$; then (c) follows.

For any $E \in \operatorname{Irr} W$ we define $a_{E} \in \mathbf{N}$ as in [8, 4.1]. Let $\tilde{a}_{E}=\nu-a_{E}+a_{E^{\dagger}}$. (d) $T_{w_{0}}^{2}=v^{2 \tilde{a}_{E}} 1: E_{v} \rightarrow E_{v}$.

This can be deduced from [8, (5.12.2)]; a closely related statement was first proved by Springer, see [4, 9.2.2].

We show:

(e) Let $E \in \operatorname{Irr} W$ and let $d \in \mathcal{D}, w \in C_{\text {min }}^{d}$. Then all eigenvalues of $T_{w}$ : $E_{v} \rightarrow E_{v}$ (in an algebraic closure of $\mathbf{Q}(v)$ ) are roots of 1 times $v^{2 \tilde{a}_{E} / d}$.

If $d$ is as in (a) then the result follows from (a) and (d). If $d=h$ then the result follows from (b) and (d). If $d, y$ are as in (c) then the result follows from (c) and the previous sentence. From the description of $\mathcal{D}$ for various types we see that if $d \in \mathcal{D}$ is not as in (a) then it is as in (c). This proves (e). (A closely related result can be found in [4, 9.2.5].)

From (e) we deduce:

(f) In the setup of $(\mathrm{e}), \operatorname{tr}\left(T_{w}, E_{v}\right)$ equals $v^{2 \tilde{a}_{E}} / d \operatorname{tr}(w, E)$; this is 0 if $2 \tilde{a}_{E} / d \notin \mathbf{Z}$. (The idea of the proof leading to (f) appeared in [8, p.320].) Using (f) and 1(a) we deduce:

(g) If $d \in \mathcal{D}, w \in C_{\text {min }}^{d}$, then

$$
N^{w, w}=\sum_{E \in \operatorname{Irr} W} \mathbf{q}^{2 \tilde{a}_{E} / d_{\operatorname{tr}}(w, E)^{2}} .
$$


In particular, we have $N^{w, w} \in \mathbf{N}[\mathbf{q}]$ and $w$ is positive.

4. Using 1(a) and the CHEVIE package [5] one can find a list of positive conjugacy classes in $W$ (assumed to be irreducible of low rank). I thank Gongqin Li for help with programming in GAP. The list of positive conjugacy classes in $W$ which are not regular elliptic for $W$ of type $E_{6}, E_{7}, E_{8}, F_{4}, G_{2}, B_{5}, B_{6}$ is as follows. (We specify a conjugacy class by the characteristic polynomial of one of its elements in the reflection representation. We denote by $\Phi_{k}$ the $k$-th cyclotomic polynomial; thus $\Phi_{2}=\mathbf{q}+1, \Phi_{3}=\mathbf{q}^{2}+\mathbf{q}+1$, etc.)

Type $E_{6}$ : none.

Type $E_{7}: \Phi_{12} \Phi_{6} \Phi_{2}, \Phi_{10} \Phi_{6} \Phi_{2}, \Phi_{10} \Phi_{2}^{3}, \Phi_{8} \Phi_{4} \Phi_{2}, \Phi_{4}^{2} \Phi_{2}^{3}$.

Type $E_{8}: \Phi_{18} \Phi_{6}, \Phi_{18} \Phi_{2}^{2}, \Phi_{9} \Phi_{3}, \Phi_{14} \Phi_{2}^{2}$.

Type $F_{4}$ : none.

Type $G_{2}$ : none.

Type $B_{5}: \Phi_{8} \Phi_{2}, \Phi_{6} \Phi_{2}^{2}, \Phi_{4}^{2} \Phi_{2}, \Phi_{2} \Phi_{4} \Phi_{6}$.

Type $B_{6}: \Phi_{10} \Phi_{2}^{2}, \Phi_{8} \Phi_{4}, \Phi_{8} \Phi_{2}^{2}, \Phi_{6} \Phi_{2}^{3}$.

In each of these examples any positive element of $W$ is elliptic; we expect this to be true in general. The example of $B_{6}$ suggests that if $W$ is of type $B_{n}$ with $2 n=4+8+\cdots+4 k$, then an element of $W$ with cycle type (4) (8) ... (4k) might be positive.

Remark. In a first version of this paper, the fourth conjugacy class listed above for type $B_{5}$ was omitted by mistake. I thank Jean Michel for pointing this out.

5. Let $\mathbf{k}$ be an algebraic closure of the finite field $F_{q}$ with $q$ elements. Let $G$ be a connected reductive group over $\mathbf{k}$ with a fixed $F_{q}$-split rational structure and whose Weyl group is $W$. Let $F: G \rightarrow G$ be the corresponding Frobenius map. For $w \in W$ let $X_{w}$ be the variety of Borel subgroups $B$ of $G$ such that $B$ and $F(B)$ are in relative position $w$, see [2, 1.3]. The finite group $G^{F}=\{g \in G ; F(g)=g\}$ acts on $X_{w}$ by conjugation. For $w, w^{\prime}$ in $W$ we denote by $X_{w, w^{\prime}}=G^{F} \backslash\left(X_{w} \times X_{w^{\prime}}\right)$ the space of $G^{F}$-orbits for the 
diagonal action of $G^{F}$ on $X_{w} \times X_{w^{\prime}}$. Now $\left(B, B^{\prime}\right) \mapsto\left(F(B), F\left(B^{\prime}\right)\right)$ induces a map $X_{w, w^{\prime}} \rightarrow X_{w, w^{\prime}}$ (denoted again by $F$ ) which is the Frobenius map for an $F_{q^{-}}$-rational structure on $X_{w, w^{\prime}}$. By $[7,3.8]$ for any integer $e \geq 1$ we have

$$
\sharp\left(\xi \in X_{w, w^{\prime}} ; F^{e}(\xi)=\xi\right)=N^{w, w^{\prime}}\left(q^{e}\right) .
$$

6. In the remainder of this paper we assume that $G$ in no.5 is simply connected and $W$ is irreducible. In the case where $w$ is a Coxeter element of minimal length of $W$, the left hand side of 5 (a) (with $w=w^{\prime}$ ) has been computed in [6, p.158]. This gives the following formulas for $N^{w, w}$.

$$
\begin{aligned}
& \text { Type } A_{n}(n \geq 1): \mathbf{q}^{2 n}+\mathbf{q}^{2 n-2}+\cdots+\mathbf{q}^{2}+1 \\
& \text { Type } B_{n}(n \geq 2): \mathbf{q}^{2 n}+2 \mathbf{q}^{2 n-2}+2 \mathbf{q}^{2 n-4}+\cdots+2 \mathbf{q}^{2}+1 . \\
& \text { Type } D_{n}(n \geq 4): \mathbf{q}^{2 n}+\mathbf{q}^{2 n-2}+2 \mathbf{q}^{2 n-4}+2 \mathbf{q}^{n-6}+\cdots+2 \mathbf{q}^{4}+\mathbf{q}^{2}+1 . \\
& \text { Type } E_{6}: \mathbf{q}^{12}+\mathbf{q}^{10}+2 \mathbf{q}^{8}+4 \mathbf{q}^{6}+2 \mathbf{q}^{4}+\mathbf{q}^{2}+1 . \\
& \text { Type } E_{7}: \mathbf{q}^{14}+\mathbf{q}^{12}+2 \mathbf{q}^{10}+4 \mathbf{q}^{8}+2 \mathbf{q}^{7}+4 \mathbf{q}^{6}+2 \mathbf{q}^{4}+\mathbf{q}^{2}+1 . \\
& \text { Type } E_{8}: \mathbf{q}^{16}+\mathbf{q}^{14}+2 \mathbf{q}^{12}+4 \mathbf{q}^{10}+2 \mathbf{q}^{9}+10 \mathbf{q}^{8}+2 \mathbf{q}^{7}+4 \mathbf{q}^{6}+2 \mathbf{q}^{4}+\mathbf{q}^{2}+1 . \\
& \text { Type } F_{4}: \mathbf{q}^{8}+2 \mathbf{q}^{6}+6 \mathbf{q}^{4}+2 \mathbf{q}^{2}+1 . \\
& \text { Type } G_{2}: \mathbf{q}^{4}+4 \mathbf{q}^{2}+1 .
\end{aligned}
$$

Let $\mathcal{N}_{G}$ be the variety consisting of all pairs $\left(g, g^{\prime}\right)$ where $g$ runs through the standard Steinberg cross section of the set of regular elements of $G$ and $g^{\prime}$ is an element in the centralizer of $g$ in $G$ modulo the centre of $G$. (This variety, introduced in [6, p.158], makes sense even if $\mathbf{k}$ is replaced by the complex numbers. It plays a role in [3] where it is called the universal centralizer.) According to [6, p.158], the number of $F_{q}$-rational points of $\mathcal{N}_{G}$ is equal to $N^{w, w}(q)$ hence it is given by the formulas above with $\mathbf{q}=q$.

7. Let $C$ be a conjugacy class of $W$. For $w \in C$, the part of weight $j$ of the $i$-th $l$-adic cohomology space with compact support $H_{c}^{i}\left(X_{w}, \overline{\mathbf{Q}}_{l}\right)$ is a direct sum $\oplus_{\rho} V_{\rho, j}^{i} \otimes \rho$ where $\rho$ runs over the unipotent representations of $G^{F}$ (up to isomorphism) and $V_{\rho, j}^{i}$ are finite dimensional $\overline{\mathbf{Q}}_{l}$-vector spaces in such a way that the $G^{F}$-action is only through the action on $\rho$ and the Frobenius action is only through an action on $V_{\rho, j}^{i}$ (where it is multiplication by $q^{j / 2} \lambda_{\rho}$ with 
$\lambda_{\rho}$ a root of 1 independent of $w, i, j$, and the parity of $j$ is independent of $w, i$, see [7, 3.9], [8]). Using the Grothendieck-Lefschetz fixed point formula, from 5 (a) we deduce for any $e \geq 1$ :

$$
N^{w, w}\left(q^{e}\right)=\sum_{i, i^{\prime}, j, j^{\prime}, \rho}(-1)^{i+i^{\prime}} \operatorname{dim}\left(V_{\rho, j}^{i}\right) \operatorname{dim}\left(V_{\rho^{*}, j^{\prime}}^{i^{\prime}}\right) q^{j e / 2} q^{j^{\prime} e / 2}
$$

where $\rho^{*}$ is the dual of $\rho$ and we have used that $\lambda_{\rho^{*}}=\lambda_{\rho}^{-1}$. This implies

$$
N^{w, w}=\sum_{i, i^{\prime}, j, j^{\prime}, \rho}(-1)^{i+i^{\prime}} \operatorname{dim}\left(V_{\rho, j}^{i}\right) \operatorname{dim}\left(V_{\rho^{*}, j^{\prime}}^{i^{\prime}}\right) v^{j+j^{\prime}} .
$$

If we assume that

(b) the $G^{F}$-modules $H_{c}^{i}\left(X_{w}, \overline{\mathbf{Q}}_{l}\right)$, dual of $H_{c}^{i^{\prime}}\left(X_{w}, \overline{\mathbf{Q}}_{l}\right)$ are disjoint for any $i, i^{\prime}$ such that $i \neq i^{\prime} \bmod 2$

then from (a) we could deduce that $N^{w, w} \in \mathbf{N}[\mathbf{q}]$. Hence if we assume further that $w \in C_{\min }, C \neq\{1\}$ it would follow that $C$ is positive.

We conjecture that, conversely, if $C$ is positive and $w \in C_{\min }$ then (b) holds. It is also likely that in this case,

(c) the $G^{F}$-modules $H_{c}^{i}\left(X_{w}, \overline{\mathbf{Q}}_{l}\right), H_{c}^{i^{\prime}}\left(X_{w}, \overline{\mathbf{Q}}_{l}\right)$ are disjoint for any $i, i^{\prime}$ such that $i \neq i^{\prime} \bmod 2$.

This disjointness property holds when $w$ is as in $\S 6$, see [6].

\section{References}

1. N. Bourbaki, Groupes et algèbres de Lie, Hermann, Paris, 1968.

2. P. Deligne and G. Lusztig, Representations of reductive groups over finite fields, Ann. Math., 103 (1976), 103-161.

3. M. Finkelberg and A. Tsymbaliuk, Multiplicative slices, relativistic Toda lattice and shifted quantum affine algebras, arxiv:1708.01795.

4. M. Geck and G. Pfeiffer, Characters of finite Coxeter groups and Iwahori-Hecke algebras, Clarendon Pres, Oxford, 2000.

5. M. Geck, G. Hiss, F. Lübeck, G. Malle and G. Pfeiffer, A system for computing and processing generic character tables for finite groups of Lie type, Weyl groups and Hecke algebras, Appl. Algebra Engrg. Comm. Comput., 7 (1996), 115-1210.

6. G. Lusztig, Coxeter orbits and eigenspaces of Frobenius, Invent. Math., 38 (1976), 101-159. 
7. G. Lusztig, Representations of finite Chevalley groups, Regional Conf. Ser. in Math., 39, Amer. Math. Soc. 1978.

8. G. Lusztig, Characters of reductive groups over a finite field, Ann. Math. Studies, 107, Princeton Univ. Press, 1984.

9. T. A. Springer, Regular elements of finite reflection groups, Invent. Math., 25 (1974), 159-193. 